Julia Rodrigues Martins Pastor dos Santos ${ }^{1}$ André Luis Mazzei Albert ${ }^{1}$ Katia Christina Leandro ${ }^{1}$

\title{
IMPORTÂNCIA DE UMA REGULAMENTAÇÃO ESPECÍFICA COM AS DEFINIÇÕES E CLASSIFICAÇÕES DOS PRODUTOS COMERCIALIZADOS COMO SUPLEMENTOS ALIMENTARES, ALIMENTOS FUNCIONAIS E NUTRACÊUTICOS*
}

Importance of a specific legislation with definitions and classifications of products marketed as food supplements, functional foods and nutraceuticals

${ }^{1}$ Instituto Nacional de Controle de Qualidade em Saúde da Fundação Oswaldo Cruz. Rio de Janeiro/RJ, Brasil.

Correspondência: Katia Christina Leandro. E-mail: kcleandro@gmail.com

Recebido: 09/11/2017. Revisão: 17/05/2018. Aprovado: 21/05/2018.

\footnotetext{
*O presente artigo teve como base a dissertação: SANTOS, Julia Rodrigues Martins Pastor dos Desenvolvimento de método de triagem na identificação de anorexígenos, estimulantes e diuréticos não declarados na rotulagem dos suplementos alimentares e emagrecedores nacionais. 2017. Dissertação (Mestrado) - Instituto Nacional de Controle de Qualidade em Saúde, Fundação Oswaldo Cruz, Rio de Janeiro/RJ, 2017.
} 


\section{RESUMO}

Este artigo trata da importância de a legislação brasileira regulamentar o termo "suplemento alimentar", a fim de se adequar às legislações internacionais e ao cenário desse mercado, marcado por constantes inovações tecnológicas e, consequentemente, uma ampla variedade de produtos. São descritas as leis e resoluções de diretoria colegiada publicadas pela Agência Nacional de Vigilância Sanitária para o registro desses produtos, que são classificados legalmente como alimentos. $\mathrm{O}$ artigo apresenta, ainda, as classificações de legislações internacionais e os casos de adulterações.

\section{Palavras-Chave:}

Alimentos Funcionais; Legislação; Nutracêuticos.

\section{ABSTRACT}

This study aims at showing how important is that the Brazilian legislation regulate the term "food supplement", in order to comply with international legislation and the market scenario characterized by constant technological innovations and, consequently, a wide variety of products. Laws and resolutions of the collegiate board are described, which have been published by the Brazilian Health Regulatory Agency for the registration of these products, legally classified as foods. This paper also describes the classifications of international laws and cases of adulteration.

\section{Keywords:}

Functional Foods; Legislation; Nutraceuticals. 


\section{Introdução}

Não existe um marco regulatório para suplemento alimentar na legislação brasileira, mesmo essa categoria sendo amplamente reconhecida pelo consumidor e adotada em outros países. Atualmente, esses produtos encontram-se classificados como alimentos em seis categorias, sendo que alguns deles podem, ainda, ser enquadrados como medicamentos específicos ${ }^{1}$.

As determinações legais visam a criar um sistema que permita que os produtos cheguem ao consumidor com qualidade e segurança. No entanto, a legislação pode levar a entendimentos diferenciados dos conceitos, como acontece com muitos suplementos que se encontram atualmente classificados como produtos à base de vitaminas e minerais ${ }^{2}$.

Neste último caso, também são divididos em: (i) suplementos vitamínicos e/ou minerais e (ii) medicamentos à base de vitaminas e minerais, de acordo com os níveis de micronutrientes oferecidos na dosagem diária recomendada ${ }^{3}$. Para esta categoria, o limite entre os conceitos de alimento e de medicamento é tênue, tornando difícil sua diferenciação principalmente pelos consumidores, que muitas das vezes desconhecem essa diferença ${ }^{4}$.

Uma regulamentação que reúna em uma única categoria todos os suplementos alimentares, que defina o que pode estar contido nesses produtos, bem como os limites máximo e mínimo de cada constituinte e as alegações que podem ser realizadas, facilitará o controle sanitário e o entendimento dos consumidores e contribuirá para o acesso a suplementos alimentares seguros e de qualidade ${ }^{5}$.

\footnotetext{
${ }^{1}$ AGÊNCIA NACIONAL DE VIGILÂNCIA SANITÁRIA - ANVISA. Suplementos alimentares: Documento de base para discussão regulatória. Disponível em: <http://portal.anvisa.gov.br/documents/219201/3322895/ Documento+de+base+sobre+regulamenta\%C3\%A7\%C3\%A3o+de+suplementos+alimentares/2e334a9ea456-48ef-981eef54f3b9029a>. Acesso em: 05 maio 2018.

${ }^{2}$ CARVALHO, Patrícia Borges de; ARAUJO, Wilma Maria Coelho. Rotulagem de suplementos vitamínicos e minerais: uma revisão das normas federais. Ciência e Saúde Coletiva, Rio de Janeiro, v. 13, p. 779-791, abr. 2008. Disponível em: <http://www.scielo.br/pdf/csc/v13s0/a28v13s0.pdf>. Acesso em: 30 maio 2011. http://dx.doi.org/10.1590/S1413-81232008000700028.

${ }^{3}$ BRASIL. Ministério da Saúde. Portaria n. 32, de 13 de janeiro de 1998. A Agência Nacional de Vigilância Sanitária aprova o regulamento técnico para fixação de identidade e qualidade de suplementos vitamínicos e ou de minerais. Disponível em: <http://bvsms.saude.gov.br/bvs/saudelegis/svs1/1998/ prt0032_13_01_1998.html>. Acesso em: 14 mar. 2019.

${ }^{4}$ ABE-MATSUMOTO, Lucile Tiemi; SAMPAIO, Geni Rodrigues; BASTOS, Deborah H. M. Suplementos vitamínicos e/ou minerais: regulamentação, consumo e implicações à saúde. Cad. Saúde Pública, v. 31, n. 7, jul. 2015. Disponivel em: <http://www.scielo.br/pdf/csp/v31n7/0102-311X-csp-31-7-1371.pdf>. https://doi. org/10.1590/0102-311X00177814.

${ }^{5}$ AGÊNCIA NACIONAL DE VIGILÂNCIA SANITÁRIA - ANVISA. Suplementos alimentares terão conjunto único de regras. Disponível em: <http://portal.anvisa.gov.br/noticias/-/asset_publisher/FXrpx9qY7FbU/ content/suplementos-alimentares-terao-conjunto-unico-de-regras/219201/pop_up?_101_INSTANCE_ FXrpx9qY7FbU_viewMode=print\&_101_INSTANCE_FXrpx9qY7FbU_languageld=pt_BR>. Acesso em: 26 mar. 2019.
} 
Primeiramente, serão tratadas as definições veiculadas nos meios de comunicação sobre os alimentos e medicamentos, como é o caso dos termos "alimento funcional" e "nutracêutico", respectivamente, para então, entrar na questão dos suplementos alimentares.

\section{Alimentos funcionais}

De acordo com o Decreto-Lei n. 986/1969, considera-se alimento

Toda substância ou mistura de substâncias, no estado sólido, líquido, pastoso ou qualquer outra forma adequada, destinada a fornecer ao organismo humano os elementos normais a sua formação, manutenção e desenvolvimento ${ }^{6}$.

Historicamente, a utilização de determinados alimentos na prevenção de doenças é considerada há milhares de anos. Há cerca de 2.500 anos, Hipócrates já recomendava o uso de alimentos para fins medicamentosos, como pode ser observado em uma de suas célebres frases: "Faça do alimento seu medicamento e do medicamento, seu alimento". Porém, o interesse pelo assunto popularizou-se somente na década de 1990, quando o termo "alimento funcional" passou a ser adotado ${ }^{7}$.

Com a intensificação das pesquisas e a ampla divulgação na mídia, o conceito de alimento funcional tornou-se mais conhecido tanto pelo público leigo quanto por pesquisadores que até então não estavam envolvidos com estudos nessa área ${ }^{8}$.

Em 1997, o Japão foi o pioneiro na produção e comercialização de alimentos funcionais, mas atualmente já não é o único, visto que vários países contam com uma legislação específica. O Brasil define esses produtos como tendo alegação de propriedade funcional e/ou de saúde e estabelece os critérios para sua utilização, bem como as normas e os procedimentos para o registro dos alimentos com tais alegações. Para obtenção do registro de um alimento com alegação de propriedades funcionais e/ou de saúde, deve ser encaminhado para a Agência de Vigilância Sanitária (Anvisa) um relatório técnico científico comprovando os benefícios e a segurança do uso desse alimento ${ }^{9,10,11}$.

\footnotetext{
${ }^{6}$ BRASIL. Decreto-Lei n. 986, de 12 de outubro de 1969. Institui normas básicas sobre alimentos. Disponível em: <http://www2.camara.leg.br/legin/fed/declei/1960-1969/decreto-lei-986-21-outubro-1969-377556publicacaooriginal-1-pe.html>. Acesso em: 22 jan. 2019.

${ }^{7}$ MONTEIRO Érica de O.; MARIN, Cláudia Talan. Alimentos funcionais. Rev. Bras. Med, v. 67, p. 10-19. 2015. IId. Ibid.

9BRASIL. Lei n. 9.782, de 26 de janeiro de 1999. Define o Sistema Nacional de Vigilância Sanitária, cria a Agência Nacional de Vigilância Sanitária, e dá outras providências. Disponível em: <http://www.planalto. gov.br/ccivil_03/LEIS/L9782.htm>. Acesso em: 22 jan. 2019.

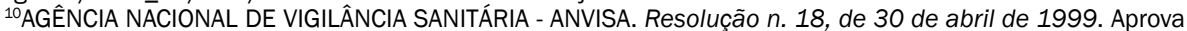
o Regulamento técnico que estabelece as diretrizes básicas para análise e comprovação de propriedades funcionais e ou de saúde alegadas em rotulagem de alimentos. Disponível em: <http://portal.anvisa.gov. br/legislacao\#/visualizar/26335>. Acesso em: 22 jan. 2019.

${ }^{11}$ AGÊNCIA NACIONAL DE VIGILÂNCIA SANITÁRIA - ANVISA. Resolução n. 19, de 30 de abril de 1999. Aprova o regulamento técnico de procedimentos para registro de alimento com alegação de propriedades funcionais e ou de saúde em sua rotulagem. Disponível em: <http://portal.anvisa.gov.br/legislacao\#/ visualizar/26345>. Acesso em: 22 jan. 2019.
} 
A indústria tem utilizado termos derivados de alimentos funcionais que muitas vezes se sobrepõem ${ }^{12}$. Frequentemente aparecem nos meios de comunicação termos como "suplementos alimentares" e "nutracêuticos", que prometem benefícios à saúde, melhor desempenho corporal e até mesmo o combate ao envelhecimento. Cada vez mais a população brasileira vem procurando ter uma alimentação saudável e, com isso, esses produtos vêm ganhando força no mercado alimentício.

Os alimentos funcionais e os nutracêuticos têm sido comumente considerados sinônimos, porém há diferenças. Os alimentos funcionais devem estar na forma de alimento comum, ser consumidos como parte da dieta e produzir benefícios específicos à saúde, tais como a redução do risco de doenças e a manutenção do bem-estar físico e mental. As substâncias biologicamente ativas presentes nos alimentos funcionais podem ser classificadas nos grupos: probióticos e prebióticos; alimentos sulfurados e nitrogenados; pigmentos e vitaminas; compostos fenólicos; ácidos graxos poli-insaturados; e fibras ${ }^{13}$. Já o termo "nutracêutico" foi introduzido em 1989, criado para tentar diferenciar os alimentos funcionais dos medicamentos. Os nutracêuticos são alimentos ou parte de alimentos que apresentam benefícios à saúde, incluindo a prevenção e/ou tratamento de doenças, podendo abranger desde nutrientes isolados até produtos herbais e alimentos processados ${ }^{14}$.

\subsection{Caracterização dos alimentos funcionais}

O termo "alimento funcional" surgiu no Japão em torno de 1980, quando o governo japonês deu início a um programa de redução de custos com seguro saúde e medicamentos - principalmente para a população que estava ficando mais velha - e procurou incentivar qualquer forma de melhorar a saúde. Foi implantado o programa Foshu (Foods for Specified Health Use, comida para uso específico de saúde), no qual se avaliavam alimentos comprovadamente benéficos para a saúde da população e que tivessem funções específicas no organismo ${ }^{15}$.

Não existe consenso em torno de uma definição oficial de alimentos funcionais. A American Dietetic Association, maior organização de alimentos e profissionais de nutrição norte-americana, classifica alimentos funcionais como alimentos convencionais, alimentos modificados (fortificados ou enriquecidos) e

\footnotetext{
${ }^{12}$ LIRA, Carlos Rogério Genari et al. Nutracêuticos: aspectos sobre segurança, controle de qualidade e legislação. Revista Brasileira de Farmácia, Rio de Janeiro, v. 90, n. 1, p. 45-49, 2009. Disponível em: <http://rbfarma.org.br/files/pag_45a49_180_nutraceuticos.pdf>.

${ }^{13}$ IKEDA, Ana Akemi; MORAES, Alexandre; MESQUITA, Gustavo. Considerações sobre tendências e oportunidades dos alimentos funcionais. Rev. P\& D Em Engenharia de Produção, v. 8, p. 40-56, 2010.

${ }^{14} \mathrm{ANJO}, \mathrm{D}$. F. C. Alimentos funcionais em angiologia e cirurgia vascular. Jornal Vascular Brasileiro, v. 3, n. 2, p. 145-154, 2004.

${ }^{15}$ FRANCO, Roberta Calhes. Análise comparativa de legislação referentes aos alimentos funcionais. 2006. Dissertação (Mestrado) - Programa de Pós-Graduação Interunidades em Nutrição Humana Aplicada PRONUT, Universidade de São Paulo, São Paulo, 2006.
} 
alimentos usados em dietas especiais ${ }^{16}$. Entretanto, nos Estados Unidos os alimentos funcionais não são oficialmente reconhecidos como uma categoria de regulamentação pela Food and Drug Administration (FDA). Já a Comissão Europeia considera alimento funcional aquele que, além dos efeitos nutricionais, tem uma ou mais funções benéficas para o organismo e que sejam relevantes para a melhoria do estado de saúde e bem-estar e/ou redução do risco de doença. Nesse contexto, alimentos funcionais não podem ser encontrados na forma de comprimidos ou cápsulas, mas sim nos alimentos e em quantidades que possam ser normalmente consumidos na dieta ${ }^{17}$.

Ao contrário dos medicamentos, os alimentos funcionais não curam doenças, e sim apresentam componentes ativos capazes de prevenir ou reduzir o risco de certas doenças. Dentre os benefícios mais investigados, estão a melhoria do sistema imunológico e a redução do risco de doenças cardiovasculares, câncer, hipertensão, diabetes, doenças inflamatórias, intestinais, certas afecções reumáticas e doença de Alzheimer ${ }^{18}$.

Em 1999, o Ministério da Saúde regulamentou o alimento funcional por meio de quatro resoluções da Anvisa (Resoluções n. $16^{19}$, n. $17^{20}$, n. 18 e n. 19) e instituiu uma Comissão Técnico-Científica de Assessoramento em Alimentos Funcionais e Novos Alimentos. Essa comissão tem por finalidade prestar consultoria e assessoramento relacionados a alimentos funcionais e novos alimentos, segurança de consumo e alegações em rótulos.

A Resolução n. 16/1999 aprova o regulamento técnico que estabelece o procedimento para registro de novos alimentos e/ou novos ingredientes. Por meio dela, o Brasil define alimentos e/ou novos ingredientes como alimentos ou substâncias sem histórico de consumo no país, ou alimentos com substâncias já consumidas que, entretanto, venham a ser adicionadas ou utilizadas em níveis muito superiores aos atualmente observados nos alimentos utilizados na dieta regular.

\footnotetext{
${ }^{16}$ HASLER, C. M.; BROWN, A. C. Position of the American Dietetic Association: functional foods. J Am Diet Assoc. v. 109, n. 4, p. 735-746, Apr. 2009. http://dx.doi.org/10.1016/j.jada.2009.02.023.PMid:19338113.

${ }^{17}$ CROWE, K. M.; FRANCIS, C. Position of the academy of nutrition and dietetics: functional foods. J Acad Nutr Diet. v.113, n. 8, p. 1096-1103, 2013. http://dx.doi.org/10.1016/j.jand.2013.06.002.

${ }^{18}$ ASSOCIAÇÃO BRASILEIRA DO COMÉRCIO FARMACÊUTICO. Afinal porque tanta discussão sobre suplementos alimentares? São Paulo: ABCFARMA, 2014. Disponivel em: <http://abcfarma.org.br/noticias/alimentosfuncionais.html>. Acesso em: 10 out. 2015.

${ }^{19}$ AGÊNCIA NACIONAL DE VIGILÂNCIA SANITÁRIA - ANVISA. Resolução n. 16, de 30 de abril de 1999. Aprova o regulamento técnico de procedimentos para registro de alimentos e ou novos ingredientes. Disponível em: <http://portal.anvisa.gov.br/legislacao\#/visualizar/26327>. Acesso em: 22 jan. 2019.

${ }^{20}$ AGÊNCIA NACIONAL DE VIGILÂNCIA SANITÁRIA - ANVISA. Resolução n. 17, de 30 de abril de 1999. Aprova o regulamento técnico que estabelece as diretrizes básicas para avaliação de risco e segurança dos alimentos. Disponível em: <http://portal.anvisa.gov.br/legislacao\#/visualizar/26333>. Acesso em: 22 jan. 2019.
} 
Considerando o interesse dos consumidores pelo uso da alimentação como determinante importante da saúde, a Anvisa estabeleceu diretrizes básicas para análise e comprovação de propriedades funcionais e/ou de saúde alegadas em rotulagem de alimentos, bem como o regulamento de procedimentos para registro de alimentos com alegação de propriedades funcionais e/ou de saúde em sua rotulagem. De acordo com a Resolução n. 19/1999, a alegação de propriedade funcional é aquela relativa ao papel metabólico ou fisiológico que o nutriente ou não nutriente tem no crescimento, no desenvolvimento, na manutenção e em outras funções normais do organismo humano; e alegação de propriedade de saúde é aquela que afirma, sugere ou implica a existência da relação entre o alimento ou ingrediente com doença ou condição relacionada à saúde. Não são permitidas alegações que façam referência à cura ou à prevenção de doenças ${ }^{21}$.

Considerando os possíveis efeitos benéficos e adversos de nutrientes e substâncias bioativas dos alimentos, no curto ou longo prazos - ou seja, a possibilidade de ocorrência de interações entre as substâncias bioativas e nutrientes ou não nutrientes no organismo com efeitos indesejáveis à saúde - a Anvisa aprovou a Resolução da Diretoria Colegiada (RDC) n. 2/2000 para avaliação de segurança, registro e comercialização de substâncias bioativas e probióticos isolados com alegação de propriedade funcional e/ou de saúde. De acordo com esse regulamento, consideram-se substâncias bioativas, além dos nutrientes, os não nutrientes que possuam ação metabólica ou fisiológica específica. A substância bioativa deve estar presente em fontes alimentares, de origem natural ou sintética, desde que comprovada a segurança para o consumo humano. São classificadas em carotenoides; fitoesteróis; flavonoides; fosfolipídeos; organossulfurados; polifenóis; e probióticos ${ }^{22}$.

Muitas substâncias bioativas vêm sendo comercializadas na forma de produtos farmacêuticos, como cápsulas, soluções, géis, pós e granulados. Esses produtos não podem ser classificados como alimentos, então o termo híbrido "nutracêuticos" (nutrientes e farmacêuticos) foi criado em 1990 pela Foundation forInnovation in Medicine, dos Estados Unidos ${ }^{23}$.

\section{Nutracêuticos}

É um termo utilizado para descrever substâncias que não são tradicionalmente reconhecidas como nutrientes (por exemplo, vitaminas e minerais), mas que

\footnotetext{
${ }^{21}$ FRANCO, Roberta Calhes. op. cit.

${ }^{22}$ AGÊNCIA NACIONAL DE VIGILÂNCIA SANITÁRIA - ANVISA. Resolução da Diretoria Colegiada n. 2, de 07 de janeiro de 2002. Aprova o regulamento técnico de substâncias bioativas e probióticos isolados com alegação de propriedades funcional e ou de saúde. Disponível em: <http://portal.anvisa.gov.br/ legislacao\#/visualizar/26836>. Acesso em: 22 jan. 2019.

${ }^{23}$ GULATI, O. M. P.; OTTAWAY, P. B. Legislation relating to nutraceuticals in the European Union with a particular focus on botanical-sourced products. Toxicology, v. 221, n. 1, p. 75-87, Apr. 2006. https://doi. org/10.1016/j.tox.2006.01.014.
} 
têm efeitos fisiológicos positivos sobre o corpo humano. O termo foi originalmente usado por Defelice em 1995, definido como "alimento ou partes de alimentos que fornecem benefícios medicinais, incluindo prevenção e/ou tratamento da doença" 24 .

No Brasil, a Anvisa não reconhece o termo "nutracêutico", e a RDC n. 2/2002 é a resolução que mais se aproxima dessa categoria. Nesse documento, encontra-se a definição de substâncias bioativas como nutrientes ou não nutrientes que possuem ação metabólica ou fisiológica específica, presentes em fontes alimentares de origem natural ou sintética, desde que comprovada a segurança para o uso humano. Não pode ter finalidade medicamentosa ou terapêutica, qualquer que seja a forma de apresentação ou o modo como é administrado.

\section{Suplementos alimentares}

Em 1994, o congresso norte-americano aprovou o Dietary Supplement Health Education Act (DSHEA), que estabeleceu que os suplementos alimentares pudessem ser tratados como alimentos, regulados de forma menos rigorosa do que medicamentos. De acordo com o DSHEA, suplementos alimentares podem conter vitaminas, minerais, ervas, aminoácidos ou outras substâncias, ou um concentrado, metabólito, constituinte, extrato ou combinação de qualquer desses ingredientes, para suplementar a dieta por aumento da ingestão total. Muitas substâncias com ações farmacológicas podem ser regularmente vendidas como suplementos alimentares nos Estados Unidos ${ }^{25}$.

A legislação brasileira não define a categoria "suplemento alimentar". Quando comercializados, fabricados ou importados no Brasil, esses produtos devem ser regularizados como medicamentos ou alimentos, de acordo com sua composição e finalidade de uso (RE n. 18/1999).

As diferentes categorias de alimentos que podem ser isentas ou não da obrigatoriedade de registro sanitário, de acordo com a Resolução da Diretoria Colegiada (RDC) n. 27/201026, são as seguintes: alimentos isentos da obrigatoriedade de registro sanitário; alimentos para controle de peso; alimentos para atletas; suplementos vitamínicos ou minerais; alimentos com obrigatoriedade de registro sanitário; alimentos com alegações de propriedade funcional e/ou de saúde; novos alimentos e novos ingredientes; substâncias bioativas e probióticos isolados.

${ }^{24}$ DEFELICE, Stephen L. The nutraceutical revolution, its impact on food industry research and development. Trends Food Sci. Technol. v. 6, p. 59-61, Feb. 1995. https://doi.org/10.1016/S0924-2244(00)88944-X.

${ }^{25}$ DA JUSTA NEVES, D. B.; CALDAS, E. D. Dietary supplements: International legal framework and adulteration profiles, and characteristics of products on the Brazilian clandestine market. Regulatory Toxicology and Pharmacology, v. 73, n. 1, p. 93-104, 2015. https://doi.org/10.1016/j.yrtph.2015.06.013.

${ }^{26}$ AGÊNCIA NACIONAL DE VIGILÂNCIA SANITÁRIA - ANVISA. Resolução da Diretoria Colegiada n. 27, de 06 de agosto de 2010. Dispõe sobre as categorias de alimentos e embalagens isentos e com obrigatoriedade de registro sanitário. Disponível em: <http://portal.anvisa.gov.br/legislacao\#/visualizar/28564>. Acesso em: 22 jan. 2019. 
Um resumo das principais categorias de alimentos que são semelhantes aos suplementos alimentares, suas definições legais e as normas reguladoras estão descritos no Quadro 1.

Os alimentos para atletas (RDC n. 18/2010) não podem ter alegação de propriedade funcional em relação a algum nutriente ou ingrediente. Esses produtos são destinados especificamente para a nutrição especial de atletas visando ao máximo desempenho em esportes com esforço muscular intenso. Outras substâncias não previstas na RDC n. 18/2010 podem ser autorizadas pela Anvisa, desde que sejam cientificamente comprovadas a segurança de uso e a eficácia da finalidade de uso (atendimento das necessidades nutricionais específicas e de desempenho no exercício).

Devido a um quadro jurídico menos restritivo, um suplemento alimentar que está disponível livremente nos Estados Unidos pode ser considerado um medicamento ou mesmo ser proibido na União Europeia e no Brasil, dando origem a um mercado clandestino. De 2007 a 2014, dados do Sistema de Alerta Rápido da União Europeia para a alimentação humana e animal registraram 929 adulterações, sendo que mais de $40 \%$ foram por ingredientes não autorizados ou medicamentos não declarados.

Quadro 1. Categorias de alimentos, definição legal e exemplos de acordo com a legislação brasileira

\begin{tabular}{|c|c|c|}
\hline Categoria & Definição legal e exemplos & Legislação \\
\hline $\begin{array}{l}\text { Alimentos para } \\
\text { atletas }\end{array}$ & $\begin{array}{l}\text { Especialmente formulados para auxiliar os atletas a } \\
\text { atender suas necessidades nutricionais específicas e } \\
\text { auxiliar no desempenho do exercício. Exemplos: whey } \\
\text { protein, creatina, aminoácidos de cadeia ramificada, } \\
\text { cafeína. }\end{array}$ & $\begin{array}{l}\text { RDC } \\
\text { n. } 18 / 2010^{27}\end{array}$ \\
\hline $\begin{array}{l}\text { Suplementos } \\
\text { vitamínicos e/ou } \\
\text { minerais }\end{array}$ & $\begin{array}{l}\text { Alimentos que servem para completar com vitaminas } \\
\text { e/ou minerais a dieta diária de uma pessoa saudável. }\end{array}$ & $\begin{array}{l}\text { Portaria } \\
\text { n. } 32 / 1998^{28}\end{array}$ \\
\hline $\begin{array}{l}\text { Alimentos com } \\
\text { alegação de } \\
\text { propriedades } \\
\text { funcionais e/ou de } \\
\text { saúde }\end{array}$ & $\begin{array}{l}\text { Alegação de propriedades funcionais: papel metabólico } \\
\text { ou fisiológico sobre o crescimento, o desenvolvimento, } \\
\text { a manutenção e outras funções normais do organismo } \\
\text { humano. Alegação de propriedade de saúde: sugere } \\
\text { existência de relação entre o alimento ou ingrediente } \\
\text { com doença ou condição relacionada à saúde. } \\
\text { Exemplos: produtos que contenham fitoesteróis, } \\
\text { omega-3, luteína e licopeno, inulina, quitosana. }\end{array}$ & $\begin{array}{l}\text { RDC } \\
\text { n. } 18 / 1999\end{array}$ \\
\hline
\end{tabular}

${ }^{27}$ AGÊNCIA NACIONAL DE VIGILÂNCIA SANITÁRIA - ANVISA. Resolução da Diretoria Colegiada n. 18, de 27 de abril de 2010. Dispõe sobre alimentos para atletas. Disponível em: <http://portal.anvisa.gov.br/ legislacao\#/visualizar/28544>. Acesso em: 22 jan. 2019.

${ }^{28}$ BRASIL. Ministério da Saúde. Portaria SVS/MS n. 32, de 13 de janeiro de 1998. Regulamento técnico para suplementos vitamínicos e/ou de minerais. Disponível em: <http://portal.anvisa.gov.br/ documents/33916/394219/PORTARIA_32_1998.pdf/551775c4-9fc2-4f62-bb62-c7ceea757476>. Acesso em: 22 jan. 2019. 
Regulação de suplementos alimentares, alimentos funcionais e nutracêuticos

Continuação

\begin{tabular}{|c|c|c|}
\hline Categoria & Definição legal e exemplos & Legislação \\
\hline $\begin{array}{l}\text { Novos alimentos } \\
\text { e/ou novos } \\
\text { ingredientes }\end{array}$ & $\begin{array}{l}\text { Alimentos ou substâncias sem histórico de consumo no } \\
\text { país, ou alimentos com substâncias já consumidas que } \\
\text { venham a ser adicionadas ou utilizadas em níveis muito } \\
\text { superiores aos atualmente observados nos alimentos } \\
\text { da dieta regular. Exemplos: óleo de peixe, lecitina de } \\
\text { soja, extrato de guaraná em cápsulas. }\end{array}$ & RDC n. 16/1999 \\
\hline $\begin{array}{l}\text { Substâncias } \\
\text { bioativas e } \\
\text { probióticos isolados } \\
\text { com alegação } \\
\text { de propriedade } \\
\text { funcional e/ou de } \\
\text { saúde }\end{array}$ & $\begin{array}{l}\text { Substância bioativa: além dos nutrientes, os não } \\
\text { nutrientes que possuem ação metabólica ou fisiológica } \\
\text { específica. } \\
\text { Probiótico: micro-organismos vivos capazes de melhorar } \\
\text { o equilíbrio microbiano intestinal, como Bifidobacterium } \\
\text { sp. }\end{array}$ & RDC n. 02/2002 \\
\hline $\begin{array}{l}\text { Alimentos para } \\
\text { controle de peso }\end{array}$ & $\begin{array}{l}\text { São os alimentos que apresentam composição definida } \\
\text { para suprir parcialmente as necessidades nutricionais } \\
\text { do indivíduo e que sejam destinados a propiciar } \\
\text { redução, manutenção ou ganho de peso corporal, } \\
\text { como os shakes. }\end{array}$ & $\begin{array}{l}\text { Portaria } n . \\
30 / 1998^{29}\end{array}$ \\
\hline
\end{tabular}

Alertas de recall de alguns produtos não regularizados têm sido emitidos pela Anvisa. Em novembro de 2014, foi emitido um alerta sobre a presença de substâncias proibidas no suplemento Mayhem. O motivo do recall foi a detecção, pelo laboratório de análises do FDA, das substâncias medicamentosas dexametasona e ciproeptadina, não declaradas no rótulo do produto. $O$ produto em questão não atende à legislação brasileira no que diz respeito à avaliação de segurança, mas é comercializado na internet e também pode ser adquirido por meio da importação direta por consumidores ${ }^{30}$. Em janeiro de 2015, a Anvisa publicou em seu site um alerta aos consumidores sobre a constatação da presença de medicamentos nos suplementos chamados B-Lipo Capsules e Slim-K Capsules, devido à detecção pelo laboratório de análises do FDA das substâncias medicamentosas lorcaserina (no produto B-Lipo Capsules) e sibutramina, desmetilsibutramina e fenolftaleína (no produto Slim-K Capsules), não declaradas nos rótulos dos produtos. Esses produtos são comercializados nos Estados Unidos como "suplementos naturais à

\footnotetext{
${ }^{29}$ BRASIL. Ministério da Saúde. Portaria SVS/MS n. 30, de 13 de janeiro de 1998. Aprova o regulamento técnico referente a alimentos para controle de peso. Disponível em: <http://portal.anvisa.gov.br/ documents/33916/394219/PORTARIA_30_1998.pdf/31b6e27f-4640-401b-9981-1b33f9834d73>. Acesso em: 22 jan. 2019.

${ }^{30}$ ALERTA sobre presença de substâncias proibidas no suplemento Mayhem. Notícias ANVISA, 18 nov. 2014. Disponível em: <http://portal.anvisa.gov.br/rss/-/asset_publisher/Zk4q6UQCj9Pn/content/alerta-sobrepresenca-de-substancias-proibidas-no-suplemento-mayhem/219201?inheritRedirect=false $>$. Acesso em: 07 nov. 2015.
} 
base de ervas para emagrecimento" e, mesmo não estando regularizados no Brasil, podem ser importados para uso pessoal ${ }^{31}$.

Diante dos relatos na literatura sobre recall dos produtos comercializados como suplementos alimentares e dos diferentes riscos que esses produtos apresentam, e levando em consideração que podem ser enquadrados em diferentes categorias, as quais misturam características de medicamentos e alimentos, gerando dúvidas nos consumidores, a Anvisa reconheceu a necessidade de rever a legislação relativa a suplementos alimentares de acordo com as características desse mercado ${ }^{32}$.

O então diretor-presidente da Anvisa, Jarbas Barbosa, em 2017, enfatizou o interesse da agência de promover uma ampla discussão sobre as novas normas e a busca pela harmonização internacional das normas de suplementos alimentares. Outra questão é a formulação de uma normatização mais flexível, com uma resolução de diretoria colegiada que traga os princípios norteadores, mas que também tenha instruções normativas que mudem e se adaptem periodicamente ${ }^{33}$. De acordo com o documento de base para discussão regulatória ${ }^{34}$, pretende-se reunir em uma única categoria de suplementos alimentares os produtos que hoje estão enquadrados em seis categorias de alimentos (Quadro 1) e uma categoria de medicamentos (medicamentos específicos sem prescrição médica).

A constante evolução científica e tecnológica na área de suplementos exige que a regulamentação dos suplementos seja tratada como um tema de atualização periódica, pois, em função de sua natureza e dinamicidade, resultam em frequentes modificações nos anexos ou listas vinculadas.

Um marco regulatório para suplementos alimentares que agrupe as diferentes categorias existentes em uma só e que acompanhe a inovação tecnológica e os avanços da ciência contribuiria para que a composição e a rotulagem dos suplementos fossem mantidas atualizadas com base nas evidências científicas, contribuindo para o controle sanitário e, consequentemente, para a proteção da saúde.

${ }^{31} A G E \hat{E N C I A ~ N A C I O N A L ~ D E ~ V I G I L A ̂ N C I A ~ S A N I T A ́ R I A ~-~ A N V I S A . ~ E q u i p e ~ T e ́ c n i c a ~ d a ~ R e d e ~ d e ~ A l e r t a ~ e ~ C o m u n i c a c ̧ a ̃ o ~}$ de Riscos de Alimentos - REALI. Atualização n. 01- REALI ALERTA n. 34/2014 - Recall dos produtos B-Lipo Capsules e Slim-K Capsules por conter as substâncias não declaradas. Disponível em: <http://www. paraiso.mg.gov.br/downloads/Recall\%20dos\%20produtos\%20B-Lipo\%20Capsules\%20e\%20Slim-K\%20 Capsules.pdf>. Acesso em: 26 mar. 2019.

${ }^{32}$ AGÊNCIA NACIONAL DE VIGILÂNCIA SANITÁRIA - ANVISA. Suplementos alimentares: Documento de base para discussão regulatória, cit.

${ }^{33}$ AGÊNCIA NACIONAL DE VIGILÂNCIA SANITÁRIA - ANVISA. Evento debate regulação de suplementos alimentares. Disponível em: http://portal.anvisa.gov.br/noticias/-/asset_publisher/FXrpx9qY7FbU/ content/evento-debate-regulacao-de-suplementos-alimentares/219201?p_p_auth=YdftBkqD\&inheritRedi rect=false. Acesso em: 26 mar. 2019.

${ }^{34}$ Id. Ibid. 


\section{Conclusão}

A partir da revisão bibliográfica realizada, observou-se que a legislação brasileira necessita de um marco regulatório referente a suplementos alimentares que possibilite a harmonização das categorias vigentes, já que se trata de uma normatização extensa, complexa e de difícil entendimento.

A regulamentação da categoria de suplementos alimentares também permitirá que a legislação nacional se aproxime da de outros países, respeitando as particularidades nacionais.

Levando-se em consideração que os consumidores de suplementos alimentares estão expostos a riscos de eventos adversos, a regulamentação da categoria de suplementos alimentares permitirá o uso racional desses produtos. Por exemplo, novos critérios de rotulagem que facilitem o entendimento da população, e até mesmo dos prescritores, e elaboração de regras sobre boas práticas de fabricação e mecanismos para monitorização dos eventos adversos relacionados ao consumo de suplementos alimentares contribuirão para a garantia da segurança e da eficácia dos produtos comercializados.

\section{Referências}

ABE-MATSUMOTO, Lucile Tiemi; SAMPAIO, Geni Rodrigues; BASTOS, Deborah H. M. Suplementos vitamínicos e/ou minerais: regulamentação, consumo e implicações à saúde. Cad. Saúde Pública, v. 31, n. 7, jul. 2015. Disponível em: <http://www.scielo.br/pdf/csp/ v31n7/0102-311X-csp-31-7-1371.pdf>. https://doi.org/10.1590/0102-311X00177814.

AGÊNCIA NACIONAL DE VIGILÂNCIA SANITÁRIA - ANVISA. Suplementos alimentares: Documento de base para discussão regulatória. Disponível em: <http://portal.anvisa.gov.br/ documents/219201/3322895/Documento+de+base+sobre+regulamenta\%C3\%A7\%C3\%A $30+d e+$ suplementos+alimentares/2e334a9e-a456-48ef-981eef54f3b9029a $>$. Acesso em: 05 maio 2018.

AGÊNCIA NACIONAL DE VIGILÂNCIA SANITÁRIA - ANVISA. Equipe Técnica da Rede de Alerta e Comunicação de Riscos de Alimentos - REALI. Atualização n. 01-REALI ALERTA n. 34/2014 - Recall dos produtos B-Lipo Capsules e Slim-K Capsules por conter as substâncias não declaradas. Disponível em: <http://www.paraiso.mg.gov.br/downloads/ Recall\%20dos\%20produtos\%20B-Lipo\%20Capsules\%20e\%20Slim-K\%20Capsules.pdf>. Acesso em: 26 mar. 2019.

ALERTA sobre presença de substâncias proibidas no suplemento Mayhem. Notícias ANVISA, 18 nov. 2014. Disponível em: <http://portal.anvisa.gov.br/rss/-/asset_publisher/ Zk4q6UQCj9Pn/content/alerta-sobre-presenca-de-substancias-proibidas-no-suplemento-ma yhem/219201?inheritRedirect=false $>$. Acesso em: 07 nov. 2015. 
ANJO, D. F. C. Alimentos funcionais em angiologia e cirurgia vascular. Jornal Vascular Brasileiro, v. 3, n. 2, p. 145-154, 2004.

ARAÚJO, Leandro Rodrigues de; ANDREOLO, Jesuíno; SILVA, Maria Sebastiana. Utilização de suplemento alimentar e anabolizante por praticantes de musculação nas academias de Goiânia-GO. Rev. Bras. Ciên. eMov., Brasília, v.10, n. 3, p. 13-18, jul. 2002. Disponível em: $<$ https://portalrevistas.ucb.br/index.php/RBCM/article/viewFile/457/482>.

ASSOCIAÇÃO BRASILEIRA DO COMÉRCIO FARMACÊUTICO. Afinal porque tanta discussão sobre suplementos alimentares? São Paulo: ABCFARMA, 2014. Disponível em: <http://abcfarma.org.br/noticias/alimentos-funcionais.html>. Acesso em: 10 out. 2015.

CARVAlHO, Patrícia Borges de; ARAUJO, Wilma Maria Coelho. Rotulagem de suplementos vitamínicos e minerais: uma revisão das normas federais. Ciência e Saúde Coletiva, Rio de Janeiro, v. 13, p. 779-791, abr. 2008. Disponível em: <http://www.scielo.br/ pdf/csc/v13s0/a28v13s0.pdf>. Acesso em: 30 maio 2011. http://dx.doi.org/10.1590/S141381232008000700028 .

CROWE, K. M.; FRANCIS, C. Position of the academy of nutrition and dietetics: functional foods. J Acad Nutr Diet. v.113, n. 8, p. 1096-1103, 2013. http://dx.doi.org/10.1016/j. jand.2013.06.002.

DA JUSTA NEVES, D. B.; CALDAS, E. D. Dietary supplements: International legal framework and adulteration profiles, and characteristics of products on the Brazilian clandestine market. Regulatory Toxicology and Pharmacology, v. 73, n. 1, p. 93-104, 2015. https://doi.org/10.1016/j. yrtph.2015.06.013.

DE ROSE, Eduardo Henrique et al. Uso referido de medicamentos e suplementos alimentares nos atletas selecionados para controle de doping nos Jogos Sul-Americanos. Rev. Bras Med Esporte, v. 12, n. 5, p. 239-242, 2006. Disponível em: <http://www.scielo.br/pdf/rbme/v12n5/03. pdf>. http://dx.doi.org/10.1590/S1517-86922006000500003.

DEFELICE, Stephen L. The nutraceutical revolution, its impact on food industry research and development. Trends Food Sci. Technol. v. 6, p. 59-61, Feb. 1995. https://doi.org/10.1016/ S0924-2244(00)88944-X.

FRANCO, Roberta Calhes. Análise comparativa de legislação referentes aos alimentos funcionais. 2006. Dissertação (Mestrado) - Programa de Pós-Graduação Interunidades em Nutrição Humana Aplicada - PRONUT, Universidade de São Paulo, São Paulo, 2006.

GULATI, O. M. P.; OTTAWAY, P. B. Legislation relating to nutraceuticals in the European Union with a particular focus on botanical-sourced products. Toxicology, v. 221, n. 1, p. 7587, Apr. 2006. https://doi.org/10.1016/j.tox.2006.01.014.

HASLER, C. M.; BROWN, A. C. Position of the American Dietetic Association: functional foods. J Am Diet Assoc. v. 109, n. 4, p. 735-746, Apr. 2009. http://dx.doi.org/10.1016/j. jada.2009.02.023.PMid:19338113. 
Regulação de suplementos alimentares, alimentos funcionais e nutracêuticos

IKEDA, Ana Akemi; MORAES, Alexandre; MESQUITA, Gustavo. Considerações sobre tendências e oportunidades dos alimentos funcionais. Rev. P \& D Em Engenharia de Produção, v. 8 , p. $40-56,2010$.

LIRA, Carlos Rogério Genari et al. Nutracêuticos: aspectos sobre segurança, controle de qualidade e legislação. Revista Brasileira de Farmácia, Rio de Janeiro, v. 90, n. 1, p. 45-49, 2009. Disponível em: <http://rbfarma.org.br/files/pag_45a49_180_nutraceuticos.pdf >.

MONTEIRO Érica de O.; MARIN, Cláudia Talan. Alimentos funcionais. Rev. Bras. Med, v. 67, p. 10-19. 2015.

Julia Rodrigues Martins Pastor dos Santos - Mestre em vigilância sanitária com ênfase na qualidade de produtos, ambientes e serviços pelo Instituto Nacional de Controle de Qualidade em Saúde da Fundação Oswaldo Cruz (INCQS/Fiocruz); graduada em Farmácia pela Universidade Federal do Rio de Janeiro (UFRJ). Rio de Janeiro/RJ, Brasil.

André Luis Mazzei Albert - Doutor em Química pela Universidade Federal do Rio de Janeiro (UFRJ); mestre em Físico-Química pela UFRJ; graduado em Engenharia Química pela Universidade do Estado do Rio de Janeiro (UERJ); professor do Programa de Pós-Graduação em Vigilância Sanitária do Instituto Nacional de Controle de Qualidade em Saúde da Fundação Oswaldo Cruz (INCQS/Fiocruz). Pesquisador associado ao Laboratório de Síntese e Análise de Produtos Estratégicos do Instituto de Química da UFRJ. Rio de Janeiro/RJ, Brasil.

Katia Christina Leandro - Doutora em Química Analítica pela Pontifícia Universidade Católica do Rio de Janeiro (PUC-Rio); graduada em Química pela Universidade Federal Fluminense (UFF); professora no Programa de Pós-Graduação em Vigilância Sanitária do Instituto Nacional de Controle de Qualidade em Saúde da Fundação Oswaldo Cruz (INCQS/ Fiocruz). Membro da Comissão de Residência Multiprofissional do INCQS/Fiocruz; membro do corpo editorial da Revista Vigilância Sanitária em Debate: sociedade, ciência \& tecnologia. Rio de Janeiro/RJ, Brasil.E-mail: katia.leandro@incqs.fiocruz.br 